\title{
Hand Gesture Recognition Using Machine Learning and the Myo Armband
}

\author{
Marco E. Benalcázar, Andrés G. Jaramillo, Jonathan \\ A. Zea, and Andrés Páez \\ Departamento de Informática y Ciencias de la Computación \\ Escuela Politécnica Nacional, EPN \\ Quito, Ecuador \\ \{marco.benalcazar, andres.jaramilloy\}@epn.edu.ec
}

\author{
Víctor Hugo Andaluz \\ Universidad de las Fuerzas Armadas, ESPE \\ Sangolquí, Ecuador \\ vhandaluz1@espe.edu.ec
}

\begin{abstract}
Gesture recognition has multiple applications in medical and engineering fields. The problem of hand gesture recognition consists of identifying, at any moment, a given gesture performed by the hand. In this work, we propose a new model for hand gesture recognition in real time. The input of this model is the surface electromyography measured by the commercial sensor the Myo armband placed on the forearm. The output is the label of the gesture executed by the user at any time. The proposed model is based on the $k$-nearest neighbor and dynamic time warping algorithms. This model can learn to recognize any gesture of the hand. To evaluate the performance of our model, we measured and compared its accuracy at recognizing 5 classes of gestures to the accuracy of the proprietary system of the Myo armband. As a result of this evaluation, we determined that our model performs better $(86 \%$ accurate) than the Myo system $(83 \%)$.
\end{abstract}

Keywords-Hand gesture recogntion; EMG; machine learning; $k$-nearest neighbor; dynamic time warping algorithm

\section{INTRODUCTION}

Electromyography (EMG) is a measure of the electrical activity in the skeletal muscles of the human body [1-3]. The anatomy and physiology of a muscle can be modeled as follows. A muscle is composed of a set of overlapping motor units. A motor unit is a set of many fibers (i.e., muscular cells) innervated by a single motor neuron. The ends of the fibers are connected to the tendons. When a muscle is at rest, each fiber has an electric potential difference of approximately $-80 \mathrm{mV}$ between its extracellular and intracellular environments. On one end, motor neurons are connected to the fibers through the so-called neuromuscular junction; whereas, on the other end, they are connected to the spinal cord. When a motor neuron fires, two intracellular action potentials (i.e., waves) propagate along each fiber from the neuromuscular junction to the tendons with constant speed and without attenuation. These waves result from the depolarization and repolarization of a fiber. Thus, the motor units of a muscle produce force and movement, both in reflex and voluntary contractions (i.e., muscular activities) [2-7].

The addition of the impulse responses of the action potentials from all the fibers of a motor unit produces the so-called motor unit action potential (MUAP). The EMG produced by the activity of a skeletal muscle can be modeled as an inter- ference pattern (i.e., linear summation) between several MUAP trains. Thus, the contribution of each motor unit to the EMG can barely be recognized. The attributes (i.e., amplitude and spectrum) of an EMG depend on several factors including thickness and temperature of the skin, thickness of the fat between the muscle and the skin, velocity of the blood flow, and location of the sensors. Factors like fatigue, aging, and neuromuscular diseases degrade muscle performance as well as EMG patterns. More details about electric models of muscles and EMG signals can be found at [2-7].

Depending on the type of sensors used, there are two types of EMG: surface and intramuscular. In surface EMG, non-invasive surface sensors are placed on the skin to record the electrical activity of the muscles under it [2, 3]. In intramuscular EMG, an invasive sensor (i.e., needle) is introduced into the muscle. In this work, we use surface EMG; hence, from here on we will use EMG to mean only surface EMG.

Numerous medical and engineering applications for EMG exist. In the medical field, EMGs are used to monitor what is occurring physiologically with respect to the nerves and the muscles in real time [2]. In engineering, applications of EMG include the development of prostheses, rehabilitation devices, and human machine interaction systems [3, 5, 7]. In most of these applications, gesture recognition plays an important role. Hand gesture recognition is the problem of identifying which gesture is performed by the hand of a given user at any moment. Hand gesture recognition has many applications, including human-machine interfaces, sign language translation, and the control of human prostheses [8-10].

A gesture recognition system based on EMG can be divided into 3 stages: data acquisition, feature extraction, and classification. Data are generally acquired in 3 ways: from non-commercial surface sensors only [8, 11-15]; from commercial sensors only, such as the Myo armband [9]; and from commercial or non-commercial sensors combined with data from variables other than EMG $[8,10]$.

In the feature extraction stage, different techniques have been applied in time, frequency, and time-frequency domains to obtain meaningful information for each gesture class to be recognized. The most common features obtained in the time domain are the mean absolute value, $n$ th-order autoregressive 
coefficients, zero crossing, length of the signal, sign of slope changes, modified mean absolute value, simple square integral, root-mean square value, sample mean and variance, log detector, average amplitude change, maximum fractal length, EMG integral, Willison amplitude, histogram, cepstral coefficients, and sample entropy [5, 7-16]. The most common features obtained in the frequency domain are the power spectrum, mean and median frequencies, frequency histogram, mean power, and spectral moments $[5,7,11,13,16]$. In the time-frequency domain, the most common technique used to analyze the EMG is the wavelet transform $[5,7]$.

The classification stage consists of determining to which class the feature vector extracted from a EMG belongs. The most frequent models used for classification are support vector machines [10-12] and neural networks [14-16]. A combination of decision trees, k-means clustering and hidden Markov models is used in [8]. A combination of support vector machines and hidden Markov models is used in [15].

For several applications, gesture recognition systems are required to function in real time. The accuracy of these systems should be comparable to those in offline systems. For a gesture recognition system to function in real-time, it has to recognize a gesture in less than $300 \mathrm{~ms}$ [17]. This is equivalent to a minimum of 3 gestures/s. Additionally, these systems usually run using limited computational resources. These requirements impose a constraint in the complexity of a recognition model. Therefore, the challenge is to design a real-time gesture recognition system that simultaneously has low computational cost and exhibits good performance.

Many publications propose offline models with high accuracy, e.g., 90\% [17], 96.7\% [14], and 98\% [13], but only few propose real-time models. For example, in [10] authors propose a real-time model with a recognition accuracy of $82.3 \%$. Therefore, the problem of real-time hand gesture recognition is still open for new approaches.

In this work, we propose a new model for real-time hand gesture recognition based on forearm EMGs measured by the commercial surface sensor the Myo armband. In section 2, we describe the main characteristics of the Myo armband and the structure of our proposed model. In section 3, we describe the experiments and then present, analyze, and compare the results of the performance of both the proprietary recognition system of the Myo armband and the proposed model. Finally, in section 4, we draw some conclusions from this work.

\section{MATERIALS AND Methods}

In this section, we describe the main characteristics of the Myo armband and then present the structure of the proposed model.

\section{A. Materials}

In this work, we used the commercial sensor called Myo armband. We chose this sensor because of its relative low cost, small size and weight, and software development kit (SDK) capabilities. The SDK allowed us to communicate our recognition model with the Myo armband very easily. The Myo is composed of 8 EMG surface dry sensors. These sensors measure the electrical activity of the muscles of the forearm at a sampling rate of $200 \mathrm{~Hz}$ (Fig. 1a) with 8 bits of resolution for each sensor. The forearm muscles are responsible for the movements of the different parts of the hand (Fig. 1b). The Myo transmits its measurements to the computer via Bluetooth. Additionally, the Myo contains an inertial measurement unit (IMU) with 9 degrees of freedom (accelerometer, gyroscope, and orientation in the $x, y$, and $z$ axes). Finally, the Myo comes with a proprietary system for recognizing 5 gestures of the hand: pinch, fist, open, wave in, and wave out (Fig. 1c).

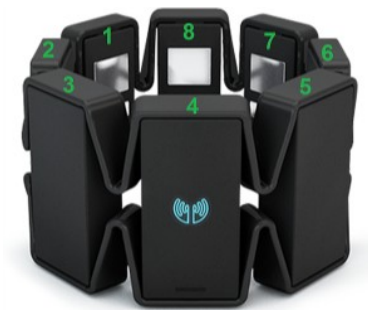

(a)
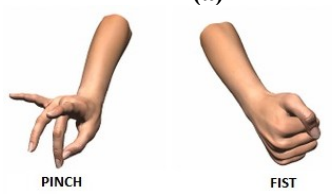

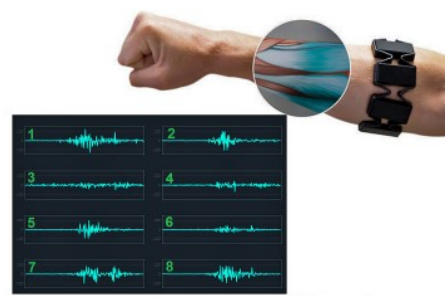

(b)
Fig. 1. Myo armband: (a) location of the surface EMG sensors on the device, (b) placement of the armband on the forearm (the sensor with the blue logo goes on the posterior part of the forearm), and (c) gestures that are recognized by the propietary software of the Myo.

\section{B. Methods}

This section describes the proposed model for real-time gesture recognition using the EMG signals and measured by the Myo armband.

1) Modeling and acquisition of EMGs: The intracellular action potentials that form an EMG occur at random intervals [1-7]. Therefore, at any moment, the EMG may be either positive or negative. Based on this context, an EMG measured during the contraction of a muscle of the forearm can be modeled by 2 zero-mean Gaussian processes [18-19]. One process is $\mathbf{S}(t, \omega) \sim \mathcal{N}\left(0, \sigma_{\text {signal }}(t), \omega\right)$ modulated by the muscle activity. The other process is an independent additive noise $\mathbf{N}(t, \omega) \sim \mathcal{N}\left(0, \sigma_{\text {noise }}, \omega\right)$. In both cases, $t$ and $\omega$ denote the instant of time and the trial number (i.e., measurement number), respectively, of the recorded EMG. The noise is assumed to be strict-sense stationary; whereas, the EMG is non-stationary (i.e., because $\sigma_{\text {signal }}$ changes with time) and independent between different EMG realizations [20].

For a given discrete instant $n \in \mathbb{Z}$ and trial number $\omega \in \mathbb{Z}^{+}$, the Myo EMG sensors return a discrete, normalized vector $\mathbf{E}(n, \omega)=\left(E_{1}(n, \omega), \ldots, E_{8}(n, \omega)\right)^{\mathrm{T}} \in[-1,1]^{8}$. The component $E_{i}(n, \omega) \in \mathbf{E}(n, \omega)$ contains the measurement returned by the $i$ th Myo EMG sensor at instant $n$ in trial $\omega$. For simplicity of notation and assuming we focus on the analysis of the sam- 
ples of a fixed $\omega$, we can write $\mathbf{E}(n)=\left(E_{1}(n), \ldots, E_{8}(n)\right)^{\mathrm{T}}$. Given the assumed nature of EMGs, the $i$ th component $E_{i}(n)$ of the vector $\mathbf{E}(n)$ is equal to $E_{i}(n)=S_{i}(n)+N_{i}(n)$, where $S_{i}(n)$ and $N_{i}(n)$ are discretized, normalized values obtained from the modulated Gaussian and noise processes, respectively, with $i=1,2, \ldots, 8$.

For obtaining feature vectors for the classification process, we define a window $W$ through which we observe $|W|=$ 200 samples from the measured EMG. Thus, at an instant $n$, we obtain the signal $\underline{\mathbf{S}}_{n}=(\mathbf{E}(n-199), \ldots, \mathbf{E}(n)) \in[-1,1]^{8 \times 200}$, where $\mathbf{E}(n-i)=\left(E_{1}(n-i), \ldots, E_{8}(n-i)\right)^{\mathrm{T}}$ and $i=0,1, \ldots, 199$. Note that 200 samples are obtained by observing the EMG through a window of $1 \mathrm{~s}$ at a sample rate of $200 \mathrm{~Hz}$. The distance between 2 consecutive observations (i.e., a stride) is 50 samples (or $0.25 \mathrm{~s}$ at a rate of $200 \mathrm{~Hz}$ ). The window length $|W|$ and the stride were determined experimentally.

2) Preprocessing: We first rectify the signal $\underline{\mathbf{S}}_{n}$ to obtain $a b s\left[\underline{\mathbf{S}}_{n}\right]$. The signal $a b s\left[\underline{\mathbf{S}}_{n}\right]$ consists of the absolute values of each element of $\underline{\mathbf{S}}_{n}$. For a given vector $\mathbf{E}(n-i) \in \underline{\mathbf{S}}_{n}$, we define $a b s[\mathbf{E}(n-i)]=\left(a b s\left[E_{1}(n-i)\right], \ldots, a b s\left[E_{8}(n-i)\right]\right)^{\mathrm{T}}$, with $i=0$, $1, \ldots, 199$. Rectification avoids a situation in which each channel of the signal $\underline{\mathbf{S}}_{n}$ averages to zero. Then, we apply a digital low-pass Butterworth filter $\boldsymbol{\Psi}$ to the signal $a b s\left[\underline{\mathbf{S}}_{n}\right]$. This filtering reduces the noise and smooths each channel (i.e., row) of $a b s\left[\underline{\mathbf{S}}_{n}\right]$. The filter $\boldsymbol{\Psi}$ has a 4 th order with a cutoff frequency of $5 \mathrm{~Hz}$ (i.e., normalized frequency of $0.05 \pi \mathrm{rad} / \mathrm{sample}$ ). As a result of filtering, we obtain the signal $\boldsymbol{\Psi}\left[a b s\left[\underline{\mathbf{S}}_{n}\right]\right]$. We chose the Butterworth filter because it has fewer design parameters than the Chebyshev and elliptic IIR filters [20]. Additionally, we preferred IIR to FIR digital filters because, to achieve a similar sharpness, IIR filters are usually less computationally expensive than FIR.

3) Feature extraction: Here, we define a feature matrix $\underline{\mathbf{X}}=\left(\mathbf{X}_{1} ; \ldots ; \mathbf{X}_{8}\right) \in \mathbb{R}^{8 \times 200}$, where its $i$ th row contains the feature vector $\mathbf{X}_{i}=\mathbf{\Psi}\left[a b s\left[E_{i}(n-199), \ldots, E_{i}(n-1), E_{i}(n)\right]\right]$, with $i=1,2, \ldots, 8$. This means that the vector $\mathbf{X}_{i} \in \underline{\mathbf{X}}$ contains the 200 samples of the $i$ th channel from the filtered and rectified EMG $\boldsymbol{\Psi}\left[a b s\left[\underline{\mathbf{S}}_{n}\right]\right]$.

4) Classification: The feature matrix $\underline{\mathbf{X}} \in \mathcal{X}$ is labeled with an element $\psi(\underline{\mathbf{X}})$ from the set $\mathcal{Y}=\{0,1, \ldots, c-1\}$, where $c$ denotes the number of classes for the classification. Here, the label 0 represents the class "no gesture" or "rest." The space of feature matrices is denoted by $\mathcal{X} \subset \mathbb{R}^{8 \times 200}$. We define the classifier $\psi: \mathcal{X} \rightarrow \mathcal{Y}$ in such a way that

$$
\psi(\underline{\mathbf{X}})=\underset{y \in\{0, \ldots, c-1\}}{\arg \max } \mathbb{P} \quad \underline{\mathbf{X}}),
$$

subject to the condition that the probability that maximizes equation (1) must be equal to or greater than a threshold $\tau \in[0,1] \forall y \neq 0$. Otherwise, we define $\psi(\underline{\mathbf{X}})=0$, which means that the classifier $\psi$ assigns $\underline{\mathbf{X}}$ to the class "no gesture."

For estimating the conditional probabilities $\mathbb{P}(Y \mid \underline{\mathbf{X}})$ with $Y \in \mathcal{Y}$, we use the $k$-nearest neighbor $(k \mathrm{NN})$ rule [21-23]. $k \mathrm{NN}$ estimates the conditional probabilities $\mathbb{P}(Y \mid \underline{\mathbf{X}})$ based on the relative frequency of the $k$ closest neighbors to $\underline{\mathbf{X}}$. To find the $k$ closest neighbors to $\underline{\mathbf{X}}$, we need a training set $\mathcal{D}$ and a distance function. The set $\mathcal{D}=\left\{\left(\underline{\mathbf{Z}}^{(1)}, Y^{(1)}\right), \ldots,\left(\underline{\mathbf{Z}}^{(N)}, Y^{(N)}\right)\right\}$ is composed of $N$ training pairs $\left(\underline{\mathbf{Z}}^{(i)}, Y^{(i)}\right)$, where $Y^{(i)}$ is the label with which the feature matrix $\underline{\mathbf{Z}}^{(i)}$ was observed. We denote the feature matrices of $\mathcal{D}$ with $\underline{\mathbf{Z}}$ instead of $\underline{\mathbf{X}}$ because for the acquisition of $\underline{\mathbf{Z}}$, we used a window length of 400 samples (i.e., $2 \mathrm{~s}$ of recording) instead of 200 samples. This window length was chosen so that the user had enough time to record each gesture during training. For the distance function, we used the dynamic time warping (DTW) algorithm with the Manhattan distance [24]. The inputs of the DTW algorithm are the feature vectors $\mathbf{X}_{i} \in \underline{\mathbf{X}}$ and $\mathbf{Z}_{i}^{(j)} \in \underline{\mathbf{Z}}^{(j)}$. The result is a real value $d t w\left(\mathbf{X}_{i}, \mathbf{Z}_{i}^{(j)}\right)$ representing the distance of the optimal alignment between the sequences $\mathbf{X}_{i}$ and $\mathbf{Z}_{i}^{(j)}$, with $i=1$, $2, \ldots, 8$ and $j=1,2, \ldots, N$. We define the distance between the feature matrices $\underline{\mathbf{X}}$ and $\underline{\mathbf{Z}}^{(j)}$ to be the sum of the DTW distances $d t w\left(\mathbf{X}_{i}, \mathbf{Z}_{i}^{(j)}\right)$ of their rows, with $i=1,2, \ldots, 8$.

5) Post-processing: In this last stage, the model returns "no gesture" if the current classification label $\psi\left(\underline{\mathbf{X}}_{n}\right)$ is the same as the previous one $\psi\left(\underline{\mathbf{X}}_{n-50}\right)$. Otherwise, if the classifications are different, the label $\psi\left(\underline{\mathbf{X}}_{n}\right)$ is returned. The matrices $\mathbf{X}_{n}$ and $\mathbf{X}_{n-50}$ contain the feature vectors extracted at the instants $n$ and $n-50$, respectively, with a stride of 50 samples. This post-processing helps us deal with the overlapping window classifications of the same gesture.

\section{RESULTS AND ANALYSIS}

In this section, we present and analyze the results from the test applied to the proposed model. We also present the results from the proprietary recognition system of the Myo armband. Based on these two results, we make comparisons.

\section{A. Performance of the Myo recognition system}

Before using the recognition system of the Myo armband, a user needs to synchronize the system by performing a wave out. By default, this system recognizes 5 classes of gestures: pinch, fist, open, wave in, and wave out. The performance of this system was evaluated based on the results of an experiment with 10 people. In this experiment, each person was guided with a video containing a sequence of 150 gestures. Each gesture lasted around $5 \mathrm{~s}$. The sequence presented in the video consists of 30 repetitions of the basic sequence: fist, open, wave in, and wave out. The order of the gestures in this basic sequence was defined randomly. In the last part of the video, users executed the 30 remaining pinches to complete the 150 gestures from the test. The pinch was put at the end because this gesture deactivates the recognition system of the 
Myo. Before the test, each person had time both to get used to the Myo and to practice the gestures for the test. All users wore the Myo on their right arm. Additionally, during the experiment, users had a visual feedback of the gestures returned by the Myo recognition system.

In Table 1, we show the confusion matrix with the results obtained from this experiment. In this table, we included the class "no gesture." This class represents all the gestures that were not detected by the Myo system. The overall recognition accuracy of the Myo was just over $83 \%$.

TABLE I. CONFUSION MATRIX OF THE MYO ARMBAND

\begin{tabular}{|c|c|c|c|c|c|c|c|}
\hline & \multicolumn{6}{|c|}{ Actual Gesture } & \multirow{2}{*}{$\begin{array}{l}\text { \%ACCURACY } \\
\text { \% ERROR }\end{array}$} \\
\hline \multirow{8}{*}{ } & & FIST & OPEN & WAVE IN & WAVE OUT & PINCH & \\
\hline & FIST & $\begin{array}{c}\mathbf{2 6 0} \\
17.33 \% \\
\end{array}$ & $\begin{array}{c}\mathbf{1 7} \\
1.13 \%\end{array}$ & $\begin{array}{c}\mathbf{3 4} \\
2.27 \% \\
\end{array}$ & $\begin{array}{c}\mathbf{1} \\
0.07 \%\end{array}$ & $\begin{array}{c}3 \\
0.2 \% \\
\end{array}$ & $\begin{array}{l}82.5 \% \\
17.5 \% \\
\end{array}$ \\
\hline & OPEN & $\begin{array}{c}\mathbf{1 3} \\
0.87 \% \\
\end{array}$ & $\begin{array}{c}\mathbf{2 6 1} \\
17.4 \% \\
\end{array}$ & $\begin{array}{c}\mathbf{1 2} \\
0.08 \%\end{array}$ & $\begin{array}{c}\mathbf{6} \\
0.4 \% \\
\end{array}$ & $\begin{array}{c}\mathbf{1} \\
0.07 \%\end{array}$ & $\begin{array}{l}89.1 \% \\
10.9 \% \\
\end{array}$ \\
\hline & WAVE IN & $\begin{array}{c}1 \\
0.07 \%\end{array}$ & $\begin{array}{c}\mathbf{0} \\
0 \% \\
\end{array}$ & $\begin{array}{c}\mathbf{2 1 1} \\
14.07 \%\end{array}$ & $\begin{array}{c}\mathbf{2} \\
0.13 \%\end{array}$ & $\begin{array}{c}\mathbf{0} \\
0 \% \\
\end{array}$ & $\begin{array}{l}98.6 \% \\
1.4 \%\end{array}$ \\
\hline & WAVE OUT & $\begin{array}{c}\mathbf{0} \\
0 \% \\
\end{array}$ & $\begin{array}{c}\mathbf{0} \\
0 \%\end{array}$ & $\begin{array}{c}\mathbf{0} \\
0 \% \\
\end{array}$ & $\begin{array}{l}\mathbf{2 5 5} \\
17 \% \\
\end{array}$ & $\begin{array}{c}\mathbf{0} \\
0 \% \\
\end{array}$ & $\begin{array}{c}100 \% \\
0 \%\end{array}$ \\
\hline & PINCH & $\begin{array}{c}4 \\
0.27 \% \\
\end{array}$ & $\begin{array}{c}1 \\
0.07 \% \\
\end{array}$ & $\begin{array}{c}\mathbf{1} \\
0.07 \% \\
\end{array}$ & $\begin{array}{c}\mathbf{2 5} \\
1.67 \% \\
\end{array}$ & $\begin{array}{c}\mathbf{2 5 9} \\
17.27 \% \\
\end{array}$ & $\begin{array}{l}89.3 \% \\
10.7 \% \\
\end{array}$ \\
\hline & NO GESTURE & $\begin{array}{c}\mathbf{2 2} \\
1.47 \%\end{array}$ & $\begin{array}{c}\mathbf{2 1} \\
1.4 \%\end{array}$ & $\begin{array}{c}\mathbf{4 2} \\
2.8 \% \\
\end{array}$ & $\begin{array}{c}11 \\
0.73 \%\end{array}$ & $\begin{array}{c}37 \\
2.47 \%\end{array}$ & $\begin{array}{c}\mathbf{0} \% \\
100 \% \\
\end{array}$ \\
\hline & $\begin{array}{c}\text { \%ACCURACY } \\
\% \text { ERROR }\end{array}$ & $\begin{array}{l}86.67 \% \\
13.33 \%\end{array}$ & $\begin{array}{l}87 \% \\
13 \%\end{array}$ & $\begin{array}{l}70.33 \% \\
29.67 \%\end{array}$ & $\begin{array}{l}85 \% \\
15 \%\end{array}$ & $\begin{array}{l}86.3 \% \\
13.7 \%\end{array}$ & $\begin{array}{l}83.07 \% \\
16.93 \%\end{array}$ \\
\hline
\end{tabular}

\section{B. Performance of the proposed model}

Each time a person wants to use the proposed model, it requires training. For this experiment, each user had to train the proposed model by executing 5 repetitions of the following 6 gestures: pinch, fist, open, wave in, wave out, and no gesture. The data for "no gesture" were acquired by asking the user to put his/her arm at rest. Each of these 30 gestures was recorded for $2 \mathrm{~s}$, creating thus a training set $\mathcal{D}$ composed of $N=30$ examples for each user. The number of training examples $N$ was selected as a tradeoff between a short training time and enough training examples to avoid overfitting [21-24]. We used $k=5$ neighbors for the $k \mathrm{NN}$ algorithm and a probability threshold of $\tau=4 / 5=0.8$. For optimal performance of the $k \mathrm{NN}$ algorithm, the value of $k$ was chosen based on the rule $k=$ ceil $\left[\log _{2}(N)\right]$, where ceil rounds toward $+\infty$. We defined $\tau=0.8$ to reduce the rate of false positives; this value of $\tau$ implies that our model returns a label from \{pinch, fist, open, wave in, wave out only if at least 4 out of the 5 labels of the closest neighbors of the feature matrix to be classified are equal. Otherwise, it returns "no gesture."

To evaluate the performance of the proposed model, again we carried out an experiment with 10 people. For this experiment, after training the model as indicated above, each person was asked to perform 30 repetitions for each class to be recognized. Each repetition was recorded for around $5 \mathrm{~s}$. For testing, users were not asked to execute the class "no gesture." Moreover, unlike in the test of the Myo system, in this experiment users did not have any feedback of the recognitions returned by our model. Because of the way our model is implemented (section II.B), for $5 \mathrm{~s}$ of recorded EMG, our model returns approximately 17 labels. The recognition was considered successful if, out of the labels returned, only one corresponded to the actual gesture and the labels remaining were "no gesture." Otherwise, the recognition was considered erroneous. For errors, if the algorithm returned 2 or more labels other than "no gesture," the error was defined as the first label different from "no gesture." In Table 2, we show the confusion matrix from this experiment. The overall accuracy of the proposed model is $86 \%$.

TABLE II. CONFUSION MATRIX OF THE PROPOSED MODEL

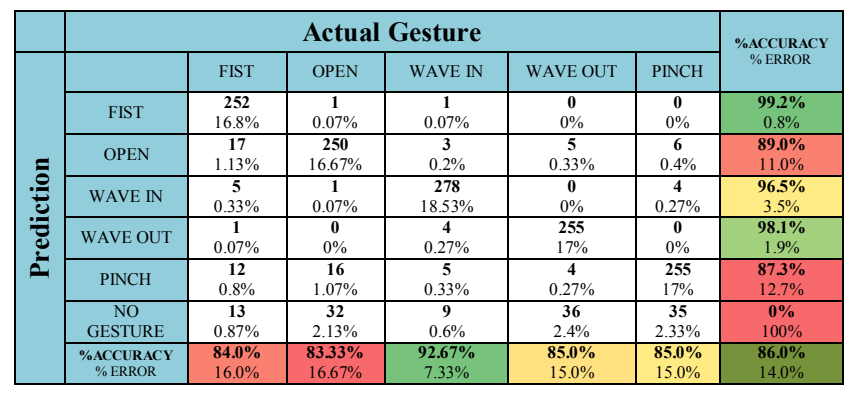

\section{Comparison of performance of Myo and proposed model}

Comparing the results in Tables 1 and 2, we see that the highest and lowest sensitivities in the proposed model occur in the classes "wave in" $(92.7 \%)$ and "open" (83.3\%). For the Myo system, the highest and lowest sensitivities were in the classes "open" (87.0\%) and "wave in" (70.3\%). Additionally, for the proposed model, the classes "fist" and "pinch" have the highest (99.2\%) and lowest (87.3\%) precision rates, respectively. Meanwhile, for the Myo system, the highest and lowest precision occurs for the classes "wave out" $(100 \%)$ and "fist" $(82.5 \%)$. The average precision of the proposed vs. the Myo system is $94.02 \%$ and $91.9 \%$, respectively. This comparison shows that the proposed model is decidedly more accurate than the Myo's proprietary system, especially taking into account that users did not have any feedback to correct their gestures while testing our model.

Because the structure of the recognition system of the Myo is not publicly available, understanding why our model performs better than the Myo is very difficult. However, during our experiments, we observed some differences in the EMG shapes when different people performed the same gesture. Therefore, we predict that training a recognition model for each user, as in our model, performs better than training a general model for all users. By training for each user, a recognition model can adapt its behavior better to the particular attributes of each user's EMG.

Regarding the computational cost of the proposed model, Fig. 2 shows a histogram of the time of processing window observations composed of 100 samples each. All the tests were carried out on a desktop computer with an Intel ${ }^{\circledR}$ Core $^{\mathrm{TM}}$ i7-3770S processor and 4GB of RAM. In this histogram, the width of each bin is $3.3 \mathrm{~ms}$. In Fig. 2, we see that most of the histogram of the processing time of each window observation is below $0.25 \mathrm{~s}$ (red line); $0.25 \mathrm{~s}$ is the time lapse we defined between 2 consecutive observations of the EMG. Therefore, our system is able to recognize, on average, 4 gestures/s. The number of classifications/s of our model is higher than the minimum recommended number of recognitions for a system to function in real time. 


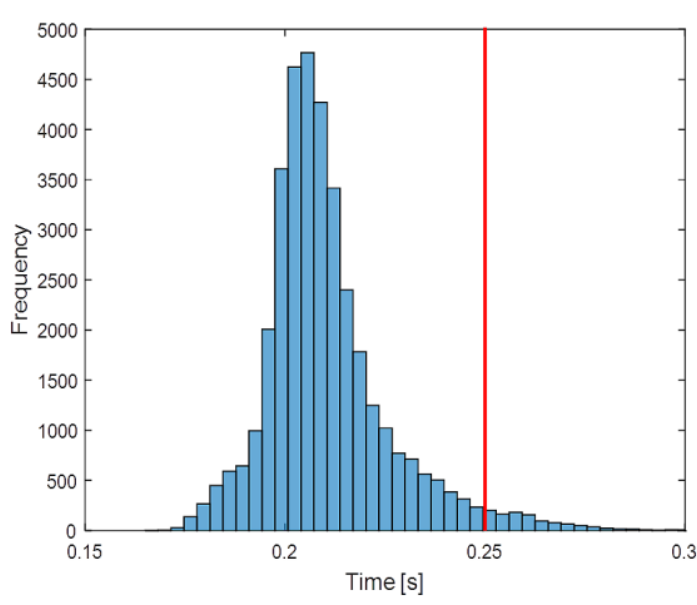

Fig. 2. Histogram of the processing time of each window observation composed of 200 samples for the proposed model.

\section{CONCLUding Remarks}

In this paper, we have presented a new model for realtime hand gesture recognition based on the EMG of the forearm. These signals are acquired using the commercial surface sensor called the Myo armband, which transmits the data via Bluetooth to the computer. The proposed model is composed of 5 stages: signal acquisition, preprocessing, feature extraction, classification, and post-processing. For the classification stage, we used the $k$-nearest neighbor rule together with the dynamic time warping algorithm.

The proposed model can learn to recognize any hand gesture through a training process in which the user performs the gestures to be recognized several times. We compared the performance of the proposed model with that of the proprietary recognition system of the Myo armband. For this comparison, we analyzed the 5 classes of gestures that the Myo armband system recognizes by default: pinch, fist, open, wave in, and wave out. As result of this comparison, we determined that our model has better recognition accuracy $(86 \%)$ than the Myo system $(83 \%)$. Additionally, we estimated that on average, our system can perform 4 recognitions/s (i.e., real-time recognition) using a desktop computer with an Intel $\AA$ Core ${ }^{\mathrm{TM}} \mathrm{i7-3770S}$ processor and $4 \mathrm{~GB}$ of RAM.

Future work includes testing the proposed model capacity to recognize gestures other than the 5 classes that the Myo system recognizes by default. Additionally, different feature extraction techniques as well as other parametric and nonparametric classification models need to be tested.

\section{ACKNOWLEDGMENT}

Authors thank Escuela Politécnica Nacional for funding this work though the research projects TELE-CEDIA-012015 and PIJ-16-13.

\section{REFERENCES}

[1] M. Haris, P. Chakraborty, and B. V. Rao, "Emg signal based finger movement recognition for prosthetic hand control," in Communication, Control and Intelligent Systems (CCIS), 2015. IEEE, 2015, pp. 194-198.

[2] L Weiss, J. Weiss, and J. Silver, "Easy EMG”, Elsevier, 2016.
[3] J. Rodriguez, A. Malanda, \& J. Navallas, "EMG modeling", INTECH Open Access Publisher, 2012.

[4] E. Petersen, "A Mathematical Model of Surface Electromyographic Measurements", Proceedings of the workshop Biosignal Processing, 2016.

[5] D. Stegeman, J. Blok, H. Hermens and K. Roeleveld, "Surface EMG models: properties and applications", Journal of Electromyography and Kinesiology, vol. 10, no. 5, pp. 313-326, 2000.

[6] M. Mordhorst, T. Heidlauf and O. Röhrle, "Mathematically modelling surface EMG signals”, PAMM, vol. 14, no. 1, pp. 123-124, 2014.

[7] M. Reaz, M. Hussain and F. Mohd-Yasin, "Techniques of EMG signal analysis: detection, processing, classification and applications", Biological Procedures Online, vol. 8, no. 1, pp. 11-35, 2006.

[8] X. Zhang, X. Chen, Y. Li, V. Lantz, K. Wang, and J. Yang, “A framework for hand gesture recognition based on accelerometer and emg sensors," IEEE Transactions on Systems, Man, and CyberneticsPart A: Systems and Humans, vol. 41, no. 6, pp. 1064-1076, 2011.

[9] D. Rodriguez, A. Piryatinska, and X. Zhang, "A neural decision forest scheme with application to emg gesture classification," in SAI Computing Conference (SAI), 2016. IEEE, 2016, pp. 243-252.

[10] C. Savur and F. Sahin, "Real-time american sign language recognition system using surface emg signal," in Machine Learning and Applications (ICMLA), 2015 IEEE 14th International Conference on. IEEE, 2015, pp. 497-502.

[11] I. Mesa, A. Rubio, J. Diaz, J. Legarda, and B. Sedano, "Reducing the number of channels and signal-features for an accurate classification in an emg pattern recognition task." in BIOSIGNALS, 2012, pp. 3848.

[12] H. Huang, T. Li, C. Bruschini, C. Enz, V. M. Koch, J. Justiz, and C. Antfolk, "Emg pattern recognition using decomposition techniques for constructing multiclass classifiers," in Biomedical Robotics and Biomechatronics (BioRob), 2016 6th IEEE International Conference on. IEEE, 2016, pp. 1296-1301.

[13] H. Ling, Y. Bo, and Z. Lina, "Clustering analysis and recognition of the emgs," in Intelligent Control and Information Processing (ICICIP), 2011 2nd International Conference on, vol. 1. IEEE, 2011, pp. 243246.

[14] R. J. Oweis, R. Rihani, and A. Alkhawaja, "Ann-based emg classification for myoelectric control," International Journal of Medical Engineering and Informatics, vol. 6, no. 4, pp. 365-380, 2014

[15] M. Rossi, S. Benatti, E. Farella, and L. Benini, "Hybrid emg classifier based on hmm and svm for hand gesture recognition in prosthetics," in Industrial Technology (ICIT), 2015 IEEE International Conference on. IEEE, 2015, pp. 1700-1705.

[16] Y. Li, Y. Tian, and W. Chen, "Multi-pattern recognition of semg based on improved bp neural network algorithm," in Proceedings of the $29^{\text {th }}$ Chinese Control Conference. IEEE, 2010, pp. 2867-2872.

[17] H. Mizuno, N. Tsujiuchi, and T. Koizumi, "Forearm motion discrimination technique using real-time emg signals," in Engineering in Medicine and Biology Society, EMBC, 2011 Annual International Conference of the IEEE. IEEE, 2011, pp. 4435-4438.

[18] P. Bonato, T. D’Alessio, and M. Knaflitz, “A Statistical Method for the Measurement of Muscle Activation Intervals from Surface Myoelectric Signal During Gait," IEEE Transactions on Biomedical Engineering, vol. 45, no. 3, pp. 287-299, 1998.

[19] L. Xu and A. Adler, "An improved method for muscle activation detection during gait," in Canadian Conference on Electrical and Computer Engineering, 2004, pp. 357-360.

[20] A. Oppenheim and R. Schafer, "Digital Signal Processing,", PrenticeHall, 1975.

[21] K. Murphy, "Machine Learning: a Probabilistic Perspective," MIT Press, 2013

[22] R. Duda, D. Stork, and P. Hart, "Pattern Classification,” Wiley 2001.

[23] L. Devroye, L. Györfi, and G. Lugosi, "A Probabilistic Theory of Pattern Recognition," Springer, 1996.

[24] M. Müller, "Information Retrieval for Music and Motion," Springer, 2007. 